\title{
CrowdLaw y democracia deliberativa: reflexiones sobre la legitimación democrática de las decisiones públicas mediante las nuevas tecnologías
}

\section{CrowdLaw and deliberative democracy: reflections on the democratic legitimation of public decisions through new technologies}

\author{
Alfonso Renato Vargas Murillo \\ Máster en Gobernanza Global y Derechos Humanos \\ Facultad de Derecho y Ciencias Políticas de la Universidad Privada de Tacna \\ Avargasm95@gmail.com
}

Enlil Iván Herrera Pérez

Maestro en Derecho

Facultad de Derecho y Ciencias Políticas de la Universidad Privada de Tacna Enlil.herrera@outlook.com

Ilda Nadia Mónica de la Asunción Pari Bedoya (it)

Máster en Cuestiones Contemporáneas en Derechos Humanos

Facultad de Derecho y Ciencias Políticas de la Universidad Privada de Tacna yldapb25@gmail.com 


\section{Resumen}

CrowdLaw surge en los últimos años como una propuesta basada en la aplicación de una serie de métodos y herramientas que utilizan como base las nuevas tecnologías para generar una interacción entre los ciudadanos y las instituciones públicas en los procesos de toma de decisiones políticas. Se plantea como hipótesis que esta particular propuesta de participación ciudadana en la toma de decisiones políticas tiene el potencial de satisfacer las condiciones que propone la democracia deliberativa para legitimar las decisiones políticas. Sin embargo, su éxito en dichos términos solo podrá suceder si viene acompañado de un proceso de derrumbamiento de la brecha y la desigualdad digital, como se produce en el caso del acceso al Internet.

\section{Palabras clave}

Crowdlaw, democracia, deliberación, tecnología, legitimidad

\section{Abstract}

CrowdLaw emerged in recent years as a proposal based on the application of a series of methods and tools that use new technologies as a basis to generate an interaction between citizens and public institutions in political decision-making processes.

It is hypothesized that this proposal for citizen participation in political decision-making has the potential to satisfy the conditions proposed by deliberative democracy to legitimize political decisions.

However, its success in terms of endings, can only happen if it is accompanied by a process to overcome the digital divide and inequality, as occurs in the case of Internet access.

\section{Keywords}

Crowdlaw, democracy, deliberation, technology, legitimacy.

\footnotetext{
Cómo citar este artículo:

Vargas, A. R., Herrera, E. I., y Pari, I. N. (2022). CrowdLaw y democracia deliberativa: reflexiones sobre la legitimación democrática de las decisiones públicas mediante las nuevas tecnologías. Revista de la Facultad de Derecho y Ciencias Políticas, 52(136), pp. 1-23. doi: https://doi.org/10.18566/rfdcp. v52n136.a01
}

Recibido: 30 de abril de 2020

Aprobado: 13 de mayo de 2021 


\section{Introducción}

El fenómeno del CrowdLaw, surge en los últimos años como una propuesta basada en la aplicación de una serie de métodos y herramientas que utilizan como base las nuevas tecnologías para generar una interacción entre los ciudadanos y las instituciones públicas en el proceso de toma de decisiones políticas. El espacio digital ofrece una serie de posibilidades novedosas y permite optimizar los tradicionales métodos de participación ciudadana en las diversas etapas del proceso legislativo e incluso en las etapas de seguimiento, evaluación y hasta de rendición de cuentas por los funcionarios públicos respecto a las políticas públicas que puedan formularse a partir de dichas normas.

La relación entre democracia deliberativa y nuevas tecnologías ha sido explorada desde diversos tópicos: la construcción del espacio público digital (Gimmler, 2001; Dahlberg, 2007; Witschge, 2004; Ess, 2018), las implicancias de la abundancia comunicativa (Ercan, et al., 2019), los peligros de la desinformación en el espacio digital (McKay y Tenove, 2020) y el rediseño de mecanismos deliberativos no diseñados inicialmente para el plano digital a la gobernanza digital (Fishkin et al. 2018). Sin embargo, el fenómeno de CrowdLaw como propuesta particular ha sido abordada recientemente desde un punto de vista teórico, en su relación con la legitimidad y calidad de la producción legislativa (Alsina y Martí, 2018), la producción de inteligencia colectiva (Noveck, 2018) y su relación con la idea de democracia abierta (Landemore, 2020); así como estudios de casos en Brasil y México (De Moraes, 2019) o España (Garnelo, 2019).

Desde esta perspectiva, planteamos como hipótesis que esta particular propuesta de participación ciudadana en la toma de decisiones políticas, denominada CrowdLaw, tiene el potencial de satisfacer las condiciones que propone la democracia deliberativa para legitimar las decisiones políticas utilizando las nuevas tecnologías. Es decir, que estas sean resultado de la interacción discursiva entre los potencialmente afectados por la decisión en condiciones de inclusión, libertad e igualdad. Sin embargo, es necesario situar esta afirmación toda vez que su éxito en dichos términos solo podrá suceder si viene acompañado de un proceso de derrumbamiento de la brecha y la desigualdad digital, como se produce en el caso del acceso al Internet. 


\section{¿Qué es CrowdLaw?}

Se puede entender como CrowdLaw la idea de que las instituciones públicas pueden funcionar mejor al fomentar la participación ciudadana a través de las nuevas tecnologías permitiendo la diversidad de fuentes de información, así como el flujo de juicios y experiencias en las etapas de formulación de leyes y políticas, con el fin de mejorar la calidad y legitimidad del resultado de dichos procesos (Alsina y Martí, 2018).

CrowdLaw surge en el año 2014 como idea de regeneración de la democracia y sus instituciones en el seno de GovLab, un centro adscrito a la Universidad de Nueva York dirigido por Beth Simone Noveck (Alsina y Martí, 2018). Una de las actividades más importantes de este grupo fue la reunión organizada con especialistas de todo el mundo que tuvo lugar en Bellagio (2018), la cual tuvo como eje la reflexión sobre las posibilidades que las nuevas tecnologías brindan para mejorar la manera en la que se toman las decisiones públicas y la manera en la que la ciudadanía participa en ellas. El producto principal de dicha reunión es un manifiesto elaborado por GovLab (2018) que reúne los aportes y los acuerdos adoptados, los que se concretan en doce principios:

1. La mejora en la forma en que se gobierna se traducirá en el incremento de confianza de la población hacia las instituciones democráticas.

2. CrowdLaw se refiere a los procesos significativos que posibilitan la participación ciudadana en cualquier etapa de los procesos de tomas de decisiones públicas (identificación de problemas, soluciones o propuestas; así como procesos de ratificaciones, implementación o evaluación, entre otros).

3. CrowdLaw se basa en la utilización de las tecnologías innovadoras para posibilitar la interacción entre los representantes, representados y funcionarios del Estado.

4. CrowdLaw puede permitir a las instituciones, a partir de un buen diseño, recabar información, opiniones y perspectivas de los ciudadanos para que estos puedan ejercer su voluntad política en los procesos de gobierno.

5. CrowdLaw, además de incrementar la confianza pública, permite el ejercicio activo de la ciudadanía, fortaleciendo la participación en las comunidades, así como la cultura democrática.

6. La participación que se puede lograr a través de un buen diseño de CrowdLaw se caracterizaría por ser eficiente, inclusiva, informada, gestionable, reflexiva y sostenible. 
7. Las instituciones gubernamentales deberían incorporar iniciativas de CrowdLaw para incrementar la legitimidad de sus decisiones a través de la participación de la ciudadanía y el incremento en la confianza pública.

8. Las instituciones públicas de todo nivel deberían implementar agendas de investigación sobre CrowdLaw y sobre la interacción entre la sociedad y las instituciones públicas.

9. La ciudadanía debe exigir y crear formas de participación, así como contribuir a partir de su conocimiento, información, experiencias y opiniones.

10.Se debe realizar un trabajo interdisciplinario con especialistas en tecnología para el desarrollo, mejora y evaluación de las plataformas de CrowdLaw con base en los diversos objetivos que se buscan alcanzar de manera ética y segura.

11.Las instituciones de gobierno deben fomentar la interacción entre las organizaciones y los diversos sectores con la finalidad de compartir las buenas prácticas.

12.Se deben implementar marcos legales que promuevan el CrowdLaw y faciliten la participación ciudadana, creando una forma de gobierno abierto, participativo y efectivo.

Estos principios, delinean en forma general lo que se pretende con esta iniciativa: mejorar la forma en la que la ciudadanía participa en las diversas etapas de los procesos de toma de decisiones públicas, particularmente a través de las nuevas TICs. Lo que se traduciría en el aumento de la calidad y la legitimidad de dichas decisiones, al generar una mayor confianza en la población hacia el gobierno. Según Alsina y Martí (2018), CrowdLaw se diferencia de otras formas de participación política por seis razones:

a. En primer lugar, al posibilitar un compromiso y una participación directa e institucionalizada que, como se verá más adelante, tiene una influencia inmediata en la legitimidad de la toma de decisiones. Dicho de otro modo, CrowdLaw tiene el potencial de impactar en la forma de ejercer el poder en un Estado, lo que está vinculado a una serie de actos que van desde la producción legislativa hasta la adopción de decisiones judiciales.

b. CrowdLaw se enfoca en la adquisición de experticia y experiencia, así como de ideas y datos objetivos debidamente estructurados, lo que permite ver a este fenómeno como una posibilidad de inteligencia colectiva.

c. Partiendo de lo anterior, se observa el potencial del CrowdLaw para el desarrollo de la legitimidad del Derecho, no bajo un concepto de "recibir opiniones”, sino de permitir una mejora en la calidad del Derecho mismo 
y las decisiones públicas. Ello es posible a través de la combinación de elementos epistémicos y procedimentales-institucionales.

d. CrowdLaw constituye la forma más sofisticada de Gobierno Abierto (Open Government), al propiciar la participación de sus ciudadanos cediendo incluso cierto control sobre la formulación de leyes y políticas. Esta característica permitiría la realización del ideal de democracia deliberativa y participativa

e. Tal como se ha descrito, el CrowdLaw facilita la participación política abierta, sin embargo, a diferencia de los enfoques tradicionales, el enfoque del CrowdLaw no se restringe a una sola etapa en el proceso de formación de leyes y políticas públicas, sino que busca que dicha participación pueda ser realizada en las diversas etapas de dicho proceso. Esto es, desde la identificación de problemas, propuestas de solución, y elaboración de proyectos, hasta la ratificación, implementación y evaluación de resultados. En tal sentido, el modelo del CrowdLaw permite una participación política holística.

f. Finalmente, esta inteligencia colectiva que busca la realización de un Gobierno Abierto que logre la maximización del concepto de democracia deliberativa y participativa, no sería posible sin el empleo de tecnología digital. Tal es así que CrowdLaw es por definición de base tecnológica. Por consiguiente, la tecnología es el ingrediente principal del que se sirve el CrowdLaw para su funcionamiento.

De esta caracterización, se puede observar que el CrowdLaw se encuentra ligado a una concepción de la democracia que busca potenciar la participación ciudadana como forma de legitimar las decisiones públicas. Sin embargo, no se refiere a cualquier tipo de participación ciudadana, como en ciertos modelos de participación poco exigente que se proponen bajo ciertas concepciones de la democracia, sino a aquella participación argumentativa que promueva una interacción dialógica, por lo que se identifica con la democracia de tipo deliberativa.

La recepción de esta propuesta ha sido global, tal como se puede observar en el siguiente cuadro: 
Tabla 1. Iniciativas de CrowdLaw en el mundo

\begin{tabular}{|l|c|c|}
\hline \multicolumn{1}{|c|}{ Región } & $\mathbf{N}^{\circ}$ & \% \\
\hline América del Sur & 17 & 16,35 \\
\hline América del Norte & 24 & 23,08 \\
\hline Europa & 41 & 39,42 \\
\hline Asia & 14 & 13,46 \\
\hline África & 4 & 3,85 \\
\hline Oceanía & 4 & 3,85 \\
\hline Total & $\mathbf{1 0 4}$ & 100 \\
\hline
\end{tabular}

Fuente: Adaptado por el autor del catálogo elaborado por GovLab (2018).

De las caracterizaciones anotadas anteriormente, la pregunta que surge es ¿cómo implementar CrowdLaw? Para implementar una propuesta es necesario establecer previamente una estrategia, y para ello es pertinente recordar -tal como fue señalado en el sexto punto característico- que CrowdLaw no es sino un modelo de Gobierno Abierto y que tiene una necesaria base tecnológica. En ese sentido, CrowdLaw emplea una estrategia de crowdsourcing para su implementación y funcionamiento, dada la compatibilidad entre las tareas que permite el crowdsourcing con aquellas que CrowdLaw requiere para su puesta en marcha.

Como lo declaran distintos autores, crowdsourcing es una convocatoria abierta para toda persona a participar en determinada tarea en línea (Brabham, 2013). Esta convocatoria agrupa diversas actividades con el fin de enriquecer a la denominada "inteligencia colectiva", ello a través de datos e información, conocimiento, 0 incluso de diversos talentos que pueden hallarse en la sociedad. De ahí la denominación de crowdsourcing, que hace alusión a un grupo abierto de personas, pero al mismo tiempo inespecífico, como fuente para, en este caso, la inteligencia colectiva.

Lo particular, como anotan Aitamurto y Landemore (2016), es que los integrantes del grupo se seleccionan ellos mismos en atención a los espacios de trabajo presentes en Internet. Partiendo de dicha idea, se tiene que el crowdsourcing tiene una base voluntaria, aunque ello no impide que puedan establecerse pagos como una forma de incentivo, en tal sentido, crowdsourcing puede ser tanto voluntario como retribuido. Además, clasifican el crowdsourcing en cuatro tipos de categorías de trabajo a las que llaman “categorías típicas", lo que supone que pueden formarse otras categorías. 
Estos cuatro tipos de categorías son: (i) microtasking, (ii) generación de ideas, (iii) búsqueda de conocimientos, y (iv) argumentación.

En cuanto a la primera categoría, microtasking, es definida por Kittur, et al. (2008) como una forma de acceder a un gran grupo de usuarios quienes realizan pequeñas tareas, pudiendo ser retribuidos con micro-pagos. Un ejemplo de un espacio de microtasking es Mechanical Turk de Amazon, la cual ofrece una contraprestación a cambio de cumplir con tareas de inteligencia humana (o HIT por sus siglas en inglés que significan "Human Inteligence Tasks”). Esta plataforma permite recopilar de forma eficiente diversos tipos de datos que un software no podría, a través de una oferta abierta sin mayores especificaciones profesionales y logrando constituirse como una herramienta de gestión de calidad eficiente.

En cuanto a la segunda teoría, el crowdsourcing se emplea para la generación de ideas, permitiendo a distintas compañías identificar soluciones para desafíos de investigación y desarrollo no resueltos (Aitamurto y Landemore, 2015). Un ejemplo, tal como proponen Aitamurto y Landemore (2016), es el servicio que presta la compañía InnoCentive, que actúa como intermediaria y brinda sus servicios a través del crowdsourcing a otras empresas que tengan problemas o necesidades por resolver, así como a usuarios que deseen resolver tales problemas o necesidades. A diferencia de la anterior categoría mencionada, en esta las tareas dadas revisten una mayor complejidad, implicando mayores incentivos, pero logrando al mismo tiempo mayores resultados eficientes al constituir una forma más sofisticada de aquella dinámica clásica apodada como "lluvia de ideas".

En cuanto a la tercera categoría, el crowdsourcing destinado a la búsqueda de conocimientos se diferencia de otros modelos colaborativos de información a gran escala como el desarrollo de software de código abierto o los modelos colaborativos tipo Wiki, donde se está frente a un modelo de pares en cuyo proceso existe una jerarquía mínima. La diferencia se encuentra en que el crowdsourcer es quien decide cuándo, dónde y cómo tendrá lugar el proceso determinado y de qué manera se utilizarán los datos aportados (Aitamurto, 2015). Como ejemplo puede citarse el actual modelo de captación de información y medios aprovechado por el periodismo. Portales como Newsflare ofrecen material audiovisual verificado y digitalmente mejorado a medios de comunicación y noticias que es aportado por usuarios a lo largo del mundo, permitiendo captar información que, de otra manera, no se alcanzaría a tener. Ello permite obtener conocimiento e información que va desde tópicos 
populares y noticias de último minuto, hasta incluso la identificación de actos de corrupción (Brito, 2008) y problemáticas sociales (Meier, 2011).

Finalmente, en cuanto a la cuarta categoría, el crowdsourcing se emplea con fines deliberativos permitiendo la exploración, evaluación y convergencia de ideas de solución sistemáticas para resolver problemas complejos a través de los aportes de grupos de interés y expertos que deseen unirse a la discusión (Klein, 2011). Aitamurto y Landemore (2016) citan como ejemplos al proyecto Deliberatorium desarrollado en MIT, y la plataforma Consider.it, a través de las cuales se permite establecer tópicos para la discusión de problemáticas complejas en las que usuarios participan dialogando pros y contras, así como proponiendo y evaluando ideas de solución bajo la moderación de los responsables en cada plataforma; lo que permite, más que una participación en un fórum, un mapeo y sistematización de argumentos.

Las iniciativas de CrowdLaw, al basarse en el crowdsourcing, adquieren diversas formas. Govlab (2018), al elaborar su catálogo con las iniciativas de CrowdLaw en diversas partes del mundo, ha elaborado criterios para clasificarlos, lo que a su vez permite identificar los diversos rasgos que caracterizan las iniciativas de este tipo. Partiendo de ello, podemos señalar que las iniciativas de CrowdLaw se caracterizan por lo siguiente: a) Se trata de iniciativas involucradas con un nivel de gobierno (nacional, regional o local); b) la participación ciudadana se produce en una o varias etapas del proceso de formulación de leyes y políticas públicas (identificación de los problemas, elaboración de soluciones, redacción, toma de decisiones, implementación y/o evaluación); c) se encarga una tarea a las personas (que contribuyan con sus ideas, opiniones, experiencia o evidencia); y, d) se sostienen en una o varias plataformas (web o móvil).

Un punto sobre el que debemos detenernos es aquel referido al momento en el que se produce la participación ciudadana a través de estos mecanismos de CrowdLaw. Según GovLab (2018) la primera etapa (identificación del problema) se refiere al establecimiento de la agenda respecto a los temas o problemas que se van a abordar, así que la participación ciudadana está orientada no solo a la identificación del problema o problemas, sino a la priorización de estos. En la segunda etapa (identificación de la solución), la participación ciudadana está orientada a delibrar y proponer enfoques para construir una solución a la problemática dada. La tercera etapa (redacción) prevé que los ciudadanos participen elaborando documentos tales como proyectos o comentarios de fórmulas legales o políticas públicas. En la cuarta etapa (toma de decisiones), 
los ciudadanos participan en los procedimientos para la aprobación de una norma o política, mostrando su respaldo a través de, por ejemplo, la votación. La quinta etapa (implementación) promueve la participación ciudadana al momento de la elaboración de planes de acción o aquellos que contribuyan a su refinamiento. En la última etapa (evaluación) los ciudadanos participarán monitoreando la puesta en práctica de las normas o políticas y su impacto en el bienestar comunitario.

Es sobre esta base que se analizarán las posibilidades y retos del CrowdLaw para mejorar la calidad y legitimidad democrática del proceso legislativo.

\section{Legitimidad política, democracia deliberativa y CrowdLaw}

La legitimidad política es vista como una virtud de las instituciones políticas y de las decisiones que estas realizan, sea que se trate de actos legislativos, políticas públicas, participación ciudadana, entre otros. Y ha sido definida en términos descriptivos como también en términos normativos.

Bajo una concepción descriptiva, Max Weber (1964) conceptualiza a la legitimidad política como aquella creencia en virtud de la cual las personas que ejercen autoridad tienen prestigio prestado al efecto. Dicha creencia, tendría como fuentes a (i) la propia tradición social; (ii) el carisma de los gobernantes; o (iii) la legalidad o la racionalidad del estado de Derecho. Esta posición conceptual sería criticada por autores como Beetham (1991), quien destaca la relevancia de las creencias de segundo orden que determinan finalmente el contenido o los términos de aquellas creencias que establecen qué es lo necesario para la legitimidad.

Por otro lado, la concepción normativa del concepto de legitimidad se enfoca en la justificación del poder político (Rawls, 1993) y el ejercicio de dicho poder, sea realizado por autoridades electas o de facto. Existirá legitimidad en sus decisiones si y sólo si otras condiciones normativas son satisfechas. De ahí que las decisiones de una autoridad política, aun siendo "autoridad”, podrían no derivar en obligaciones legítimas a imponerse sobre los gobernados.

Desde este punto de partida, Jeremy Waldron (2005) argumenta que la legitimidad política se encuentra referida a la justificación que el poder político 
pueda tener en cada persona a la cual se pretenda vincular por medio del ejercicio de dicho poder político. En todo ello, la democracia juega un papel trascendental como un componente necesario para la legitimidad política (Buchanan, 2002). En tal sentido, es necesario enfocarse en los procedimientos que alimentan la legitimidad democrática (Christiano, 1996). Respecto a ello, diversas teorías y puntos de vista han sido trazados, de los cuales se destaca la "democracia deliberativa", como un modelo de política propuesto en el que la legitimidad democrática depende de un proceso deliberativo e intersubjetivo de justificación al que acceden y participan quienes sean afectados (o potencialmente afectados) por un acto o medida política determinada (Gutmann y Thompson, 2004).

La democracia deliberativa es una teoría normativa de la democracia que se basa en el ideal de diálogo como presupuesto para la toma de decisiones legítimas. Desde su origen en el siglo pasado, diversos autores han generado aportes y a la vez han surgido discusiones (por lo tanto, también desacuerdos) sobre los elementos que componen esta teoría de la democracia, como son los sujetos que deben participar de la deliberación, las condiciones en las que se debe la deliberación, el tipo de deliberación, los mecanismos para institucionalizar la democracia deliberativa, etc. Pese a ello, podemos identificar esfuerzos por dotar de un contenido mínimo a la democracia deliberativa.

El contenido mínimo de la democracia deliberativa, en términos de Elster (1998) comprende a primera vista dos dimensiones: el elemento democrático y el elemento deliberativo. Mientras la dimensión democrática implica un proceso colectivo para la toma de decisiones que exige la participación de los potencialmente afectados por la medida que se va a tomar (directamente o a través de sus representantes), la dimensión deliberativa implica que la decisión a la que se va a arribar debe fundamentarse en los argumentos esgrimidos por los participantes. Gutmann y Thompson (2004) descomponen el ideal democrático deliberativo en cuatro caracteres: a) el intercambio mutuo de razones para la cooperación social, b) la publicidad y accesibilidad de las razones esgrimidas en dicho proceso para los afectados, c) la finalidad de la deliberación debe ser una medida política vinculante durante un periodo determinado y d) la provisionalidad de los principios morales y políticos, así como las medidas que resulten de ellas.

De esta manera, la propuesta normativa de la democracia plantea, siguiendo a Cohen (1997), un ideal sobre la asociación democrática en el cual los ciudadanos argumentan y razonan públicamente como iguales respecto a 
las normas básicas de esa asociación, comprometiéndose con el razonamiento público como forma de resolución colectiva de conflictos, legitimándose dichas normas en tanto promuevan la deliberación libre y pública.

Para Thompson (2019) las teorías que se adscriben a la corriente "deliberativista" comparten en su núcleo la exigencia de ciudadanos y de representantes de ofrecer razones que justifiquen sus posturas políticas y respondan a las de otros a propósito de la promulgación de leyes que, como resultado, serán vinculantes a ambos. Esta postura, se encuentra en contraposición con aquellas que no contemplan las exigencias de justificación ante los ciudadanos respecto a las decisiones que se van a adoptar, aquellas que fundamentan las prácticas políticas de forma exclusiva en el poder, el interés, la agregación de preferencias, 0 en la competencia.

Tabla 2. Fuentes de legitimación de los modelos de democracia

\begin{tabular}{|l|l|}
\hline Modelos de democracia & \multicolumn{1}{c|}{ Fuente de legitimación } \\
\hline Populista (Laclau) & $\begin{array}{l}\text { El líder político ungido por la masa popular toma las } \\
\text { decisiones sobre las cuestiones públicas con la finalidad de } \\
\text { articular las demandas sociales que no han sido satisfechas. }\end{array}$ \\
\hline Comunitarista (MacIntyre) & $\begin{array}{l}\text { La democracia se define a partir de la idea de bien común } \\
\text { (o al menos a partir de una interpretación comprehensiva } \\
\text { de este). }\end{array}$ \\
\hline Agonista (Mouffe), & $\begin{array}{l}\text { La lucha entre facciones políticas es la práctica imperan- } \\
\text { te en la democracia, producto de la dimensión agonista } \\
\text { inherente a las relaciones humanas, lo que imposibilita el } \\
\text { consenso. }\end{array}$ \\
\hline $\begin{array}{l}\text { Agregacionista pluralista } \\
\text { (Dahl) }\end{array}$ & $\begin{array}{l}\text { Las decisiones son el resultado de la negociación entre gru- } \\
\text { pos de interés que pugnan en condiciones de igualdad por la } \\
\text { influencia en dichas decisiones. }\end{array}$ \\
\hline $\begin{array}{l}\text { Agregacionista elitista } \\
\text { (Schumpeter) }\end{array}$ & $\begin{array}{l}\text { Las decisiones deben tomarlas un grupo de ciudadanos } \\
\text { que se encuentran en mejores condiciones respecto a su } \\
\text { capacidad y preparación para decidir sobre las cuestiones } \\
\text { públicas. }\end{array}$ \\
\hline Deliberativa (Habermas) & $\begin{array}{l}\text { La legitimidad de las decisiones deriva de un procedimiento } \\
\text { de toma de decisiones de carácter comunitario donde debe } \\
\text { estar asegurada la igual libertad política de los participantes } \\
\text { (los ciudadanos) y su autonomía, los cuales deben tener } \\
\text { la posibilidad de participar discursivamente e influenciar } \\
\text { en la formación de la voluntad política de la autoridad que } \\
\text { ejecutará la decisión resultante. }\end{array}$ \\
\hline
\end{tabular}

Fuente: Elaborada por el autor a partir de Giuffré (2018) 
El paso del tiempo y el entrecruzamiento de trabajos de corte teórico, así como de investigaciones empíricas, han producido un proceso de "maduración" del ideal democrático deliberativo. Bohman (2016) plantea que la democracia deliberativa ha dado paso a una teoría de corte realista, lo que permite mantener despierto el interés sobre su viabilidad y los esfuerzos por innovar desde este campo. De esta manera, para el autor, una teoría de la democracia deliberativa debe poseer tres elementos:

[...]complejos procedimientos morales y epistémicos de justificación; comprender la importancia de las restricciones históricas y sociales de la democracia, tales como prácticas colectivas en curso dentro de una estructura constitucional; y considerar las restricciones y oportunidades que plantean las condiciones sociales y los escenarios deliberativos más grandes. (p. 137)

Es así que, cuando planteamos analizar los mecanismos y herramientas agrupadas bajo el rótulo CrowdLaw, necesariamente tendremos que hacer referencia a un contexto en el que se produce y la disponibilidad de los medios que posibilitan la participación de la ciudadanía en las diversas etapas de la formulación de normas o políticas. De esta manera, las condiciones de legitimidad esbozadas por los teóricos de la democracia deliberativa para analizar mecanismos como el referéndum, las audiencias públicas, los presupuestos participativos, entre otros, deben situarse en un nuevo contexto de participación: el espacio público digital.

Se destaca, entonces, la importancia de la evaluación de procesos políticos. Uno de ellos, y en el cual se enfoca el presente artículo, es el proceso legislativo. Al respecto, si bien no existe una postura uniforme respecto a los criterios para evaluar la calidad del proceso legislativo, Alsina y Martí (2018) afirman que, en general, se pueden encontrar los siguientes criterios:

a. La apertura y transparencia del proceso de toma de decisiones

b. La responsabilidad de aquellos que toman las decisiones por el resultado y desempeño en el proceso

c. La inclusión de las perspectivas y las voces que se han oído en el proceso de toma de decisiones

d. La calidad deliberativa del proceso de toma de decisiones

e. El plazo de tiempo adecuado en el que se toman dichas decisiones.

f. La involucración de expertos

g. La implementación de evidencia científica durante el proceso, así como la 
comparación de alternativas basadas en un análisis de costo-beneficio

h. La necesidad y/o oportunidad de las decisiones en las circunstancias correspondientes

i. La claridad y simpleza de las decisiones tomadas

j. La coherencia interna y consistencia de las decisiones

k. La congruencia de las decisiones con el sistema legal o conforme a ley

l. La proporcionalidad de las decisiones (v.gr., al minimizar la coerción)

m. La efectividad de las decisiones, es decir, su capacidad para producir los resultados perseguidos por tales decisiones

n. La eficacia de las decisiones, es decir, su capacidad de ser efectivamente ejecutadas por las autoridades si las personas no las obedecen

o. La existencia de una adecuada evaluación ex post del impacto

Corresponde, entonces, dialogar respecto a las posibilidades y retos de esta relación, entre el CrowdLaw y la democracia deliberativa como ideal que pretende la realización del concepto de legitimidad política no sólo en el plano teórico, sino en el práctico, sobre todo al momento de satisfacer las exigencias de igualdad y libertad como valores que sostienen el procedimiento deliberativo.

Alsina y Martí (2018) desarrollan en cuatro puntos la relación existente entre el concepto de legitimidad política desde la concepción deliberativa democrática y los procesos legislativos donde intervienen las iniciativas de CrowdLaw:

1. En primer lugar, existe una relación de dependencia entre legitimidad política y proceso legislativo, de ahí que diversos criterios de evaluación de los procesos legislativos son coincidentes con los principios de legitimidad democrática. La pretensión del CrowdLaw, al respecto, es precisamente optimizar la legitimidad política y los procesos legislativos.

2. En segundo lugar, en cuanto a los resultados de los procesos políticos y legislativos, pese a que el CrowdLaw no pretende resolver la discusión sobre una estándar de justicia completo para la evaluación de la calidad de dichos resultados, sí propone fortalecer otros componentes de calidad menos problemáticos -en términos de discusión intersubjetiva- tales como la conformidad legal, la claridad y simplicidad de las decisiones, la necesidad u oportunidad, así como su eficacia y eficiencia.

3. En tercer lugar, se hace la salvedad de que, pese a que el CrowdLaw no pretende resolver la discusión sobre un estándar de justicia, tampoco se pretende abandonar dicho ideal valorativo. La identificación del estándar 
de corrección de las decisiones públicas y el respeto de dicho estándar es posible de ser realizado mediante los procedimientos del CrowdLaw, aprovechando la inteligencia colectiva de la cual se alimenta.

4. Finalmente, si bien el trabajo de establecer estándares de calidad es complejo y no ha sido resuelto del todo, CrowdLaw se preocupa por los estándares de calidad tanto de los procedimientos de toma de decisiones públicas como también de los resultados mismos. En tal sentido, dicha preocupación se ha traducido en un compromiso de abordar tales puntos como tópicos trascendentales de investigación

De esta manera, podemos afirmar que a través del CrowdLaw, los procesos políticos, y en particular los procesos legislativos, ganan legitimidad democrática al estrechar las distancias entre los representantes y los representados, interviniendo en las diversas etapas del proceso de formulación de las leyes y políticas. Sin embargo, para que esto sea coherente con a las exigencias de la democracia deliberativa, la participación ciudadana no puede ser cualquier tipo de participación, sino que debe tratarse de una participación de tipo dialógica.

La ventaja del CrowdLaw, desde la perspectiva de la democracia deliberativa, es que plantea que la participación ciudadana tiene como una de sus finalidades primordiales la producción de inteligencia colectiva, la cual, tal como la entiende Levy (2004), es:

Inteligencia repartida en todas partes, valorizada constantemente, coordinada en tiempo real, que conduce a una movilización efectiva de las competencias. (...) el fundamento y el objetivo de la inteligencia colectiva es el reconocimiento y el enriquecimiento mutuo de las personas (p. 19).

De esta manera, la inteligencia colectiva se caracteriza en primer lugar por considerar a la producción de conocimiento como un proceso de carácter discursivo intersubjetivo, una actividad compartida que supone un esfuerzo de entendimiento entre los individuos. Por lo que, se identifica como un potencial legitimador en términos de la democracia deliberativa, en cuanto establece que los ciudadanos participan a través de sus discursos interactuando entre ellos y con los miembros de las instituciones en los procesos de formulación de las leyes y políticas. 


\section{Los retos del CrowdLaw frente a la desigualdad en el acceso a Internet}

Noveck (2018) señala que, CrowdLaw tiene el potencial para poner en agenda problemas como son las desigualdades estructurales que pueden haber sido ignoradas por las élites políticas a lo largo del tiempo. Sin embargo, se anotó que en algunos casos su uso podría incrementar las desigualdades respecto a personas con menor grado de instrucción; generando incluso nuevas situaciones de exclusión. Esto fue advertido respecto al uso de estas herramientas para diseñar políticas en la Unión Europea (Sgueo, 2020). Asimismo, se advirtió que su diseño tendía a atraer personas con mayor grado de educación y con experticia en tecnología. Tal fue el caso de vTaiwan, el cual sirvió muy bien para analizar el futuro de servicios como Uber, mas generaba serias dudas sobre si tendría la misma capacidad de éxito tratando cuestiones relacionadas con la desigualdad social (Noveck, 2018).

Al respecto, resulta necesario revisar si esas carencias estructurales pueden influir en la forma en la que los ciudadanos participan en las cuestiones públicas y su eficacia al momento de incrementar la legitimidad de los procesos de toma de decisiones. Máxime si se analiza a la luz de un ideal regulativo que exige el cumplimiento (en la medida de lo posible) de determinadas condiciones.

La democracia deliberativa propone un criterio mixto de legitimidad, en tal sentido se admite que, además del procedimiento deliberativo democrático, existen ciertas cuestiones sustantivas que posibilitan e incrementan la calidad de dicho procedimiento. Sin embargo, esto ha generado una situación problemática, denominada como la "paradoja de las precondiciones de la democracia deliberativa” (Nino, 1996). Esta paradoja consiste en que, mientras la legitimidad de decisiones del procedimiento surge de la deliberación democrática, la inclusión de elementos sustantivos como precondiciones del procedimiento de deliberación reduce el espacio de deliberación. Martí (2011) reconoce que esta paradoja conduce a un "callejón sin salida”, sin embargo, pese a su inevitabilidad, existen métodos como el del "equilibrio reflexivo" propuesto por Nino para afrontar sus consecuencias. Es decir, encontrar el equilibrio entre "una mayor realización de las precondiciones de la democracia y un mayor rango de decisiones democráticas admisibles” (Martí, 2011, p. 51).

Entre los derechos a priori generalmente aceptados por los teóricos de la democracia deliberativa, y que otorgan el elemento sustancial en su criterio 
de legitimidad, encontramos al derecho a la igualdad. Esto ha llevado a que, debido a la situación advertida por Waldron (2005) a cerca del desacuerdo respecto al contenido sobre los derechos presente en las sociedades, coexistan diversas versiones sobre los alcances de este derecho, limitándose en algunos casos a una mera concepción restringida de la igualdad, entendida como igualdad política, y en otros extendiéndose y conectándola con la satisfacción de derechos económicos, sociales y culturales.

Nino (1996) advirtió sobre la importancia de mantener una visión más amplia, señalando que a partir de la comprensión de que los derechos pueden ser violados por acción y omisión, los derechos sociales "son la extensión natural de los derechos individuales” (p. 192). Es así como el autor sostiene que las precondiciones de libertad e igualdad no solo peligran frente al uso de las amenazas o el uso de la violencia, sino también frente al hecho de no otorgar de manera igualitaria los medios que posibilitan la participación efectiva en los procesos deliberativos de toma de decisiones.

Uno de los ejemplos que cita Nino, grafica uno de los principales problemas que pueden enfrentar las iniciativas de CrowdLaw: el acceso a los medios en los cuales se produce la deliberación pública. Así, en palabras de Nino (1996) “[el] derecho de la libertad de expresión, que es por supuesto un derecho a priori, no requiere sólo de ser libre de censura sino también de tener acceso positivo a los medios para comunicarse con otros” (p. 193). En este sentido, para el fenómeno en estudio, conviene prestar atención a contextos caracterizados por la exclusión institucionalizada, la marginalización histórica de ciertos grupos, las desigualdades estructurales, etc., toda vez que consideramos que esas situaciones reducen la calidad epistémica y la legitimidad del proceso de deliberación para la toma de decisiones públicas. Por esta razón la problemática de la desigualdad relacionada con el acceso al Internet resulta ser uno de los primeros obstáculos para los objetivos de legitimación de las decisiones políticas a través de la propuesta de CrowdLaw.

Si bien el presente trabajo no pretende realizar un análisis exhaustivo de la eficacia del CrowdLaw en las diversas partes del globo, podemos advertir que cualquier análisis que se realice sobre dichas iniciativas, debe situarse geográficamente.

Uno de los mayores retos que deben enfrentar las iniciativas de CrowdLaw es la brecha digital existente en países en desarrollo, en particular la brecha sobre el acceso al Internet (Tello Arista, 2016). Con la finalidad de ilustrar con 
mayor facilidad esta problemática, podemos cruzar los datos referidos a la penetración de Internet en las diversas regiones del mundo con las iniciativas de CrowdLaw. Al respecto, Internet World Stats provee los siguientes datos:

Gráfico 1. Índice de penetración de Internet por regiones geográficas

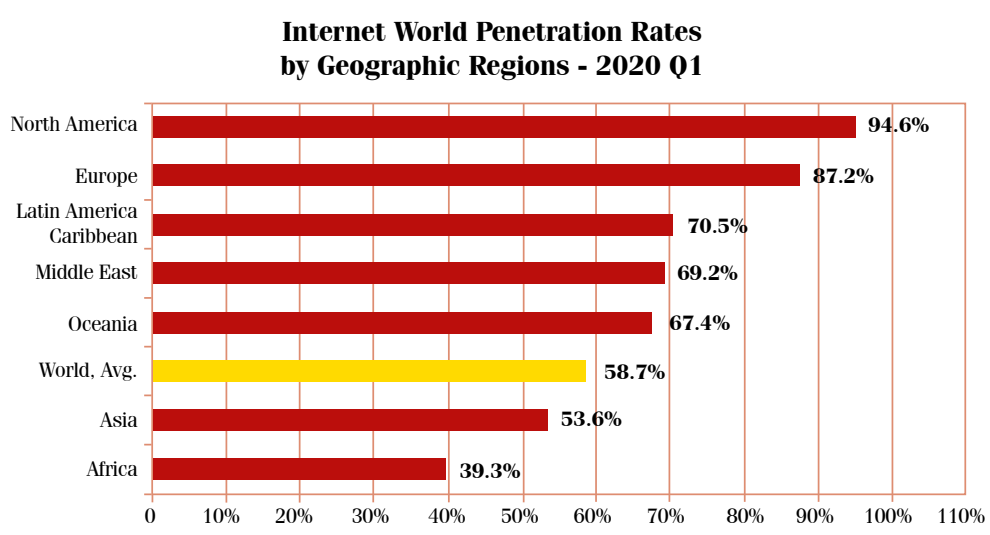

Fuente: Cuadro obtenido de Internet World Stats (2020) con datos al 03 de marzo de 2020

Del cuadro podemos observar que la presencia de iniciativas de CrowdLaw es más alta en regiones donde se posee un índice más alto de penetración de Internet (el caso de 23,08\% en Norteamérica y 39,42\% Europa) que en regiones donde el índice es inferior (3,85\% en África y 13,46\% Asia). Por lo que, si CrowdLaw tiene como objetivo mejorar la calidad de los procesos políticos y con ello contribuir a la construcción de democracias más robustas en términos de participación ciudadana, su potencial se ve disminuido (momentáneamente) frente a la falta de condiciones que permiten a los ciudadanos desarrollarse, situación que puede reflejarse en la falta de acceso a servicios como el Internet. Tal es el caso de países como Colombia, donde en el año 2019, solo 21,7 millones de personas contaban con acceso a Internet, mientras que, más de la mitad del país, 23,8 millones de colombianos, no contaban aún con este servicio (MINTIC, 2019). En un año, el número de usuarios de Internet aumentó solamente en un 2.9\% (Rosgaby. 2020).

Estas desigualdades se pueden observar de manera global en el siguiente gráfico: 
Gráfico 2. Evolución del uso del Internet en el contexto mundial

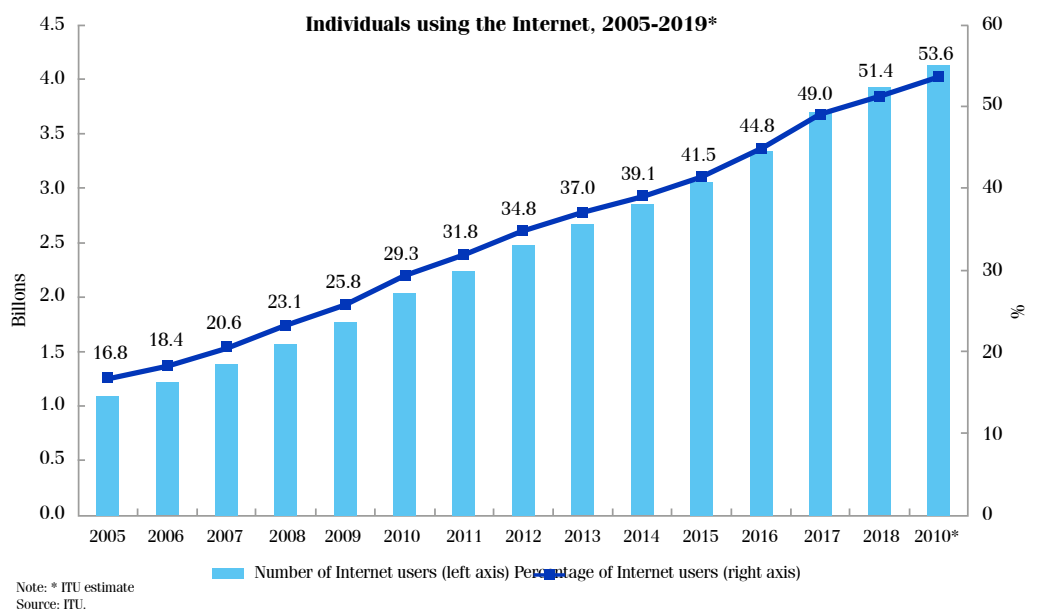

Fuente: Cuadro obtenido de la Unión Internacional de Telecomunicaciones (UIT) (2020) elaborado con datos obtenidos a fines del año 2019.

Según la UIT (2020), el 53.6\% de la población global utilizan Internet, es decir, tan solo un poco más de la mitad de la población mundial. Además, las desigualdades referidas al acceso a este servicio se evidencian cuando, según datos proporcionados por la entidad, encontramos que los usuarios en países desarrollados corresponden al $87 \%$ frente a solo el $47 \%$ de los usuarios en países en desarrollo.

Por otro lado, el perfil de los usuarios de Internet es el siguiente: hombre, residente en ciudades y joven. Por lo que la brecha digital incluye barreras en términos de edad, género, discapacidades, estatus socioeconómico y geografía. Cuando se advirtió en la ONU (2011) que el acceso al Internet debería considerarse como un Derecho Humano, partieron de la idea de que la obligación de los Estados de garantizarlo se explica por su relación que tiene con otros derechos, no solo a la libertad de expresión, sino derechos sociales como el derecho a la salud, la educación y el trabajo. Estos derechos están íntimamente vinculados con la existencia de políticas públicas ligadas al desarrollo e inclusión social, con las que se puede hacer frente a las desigualdades sociales y el problema de la inequidad (Hopenhayn, 2006). 
La pregunta que surge a continuación es ¿cómo afectan dichas desigualdades a los objetivos del CrowdLaw de incrementar la legitimidad de las decisiones públicas a través de Internet? Desde la óptica de la democracia deliberativa, podemos advertir que los retos que deben enfrentar las propuestas del CrowdLaw que utilicen como plataforma el Internet, son los siguientes:

- El derecho al acceso al Internet surge como una precondición de la democracia deliberativa referida a los procesos de toma de decisiones que se dan a través de mecanismos de CrowdLaw

- Las desigualdades sociales se traducen al plano digital en la forma de barreras que forman la brecha digital

- El acceso en condiciones de desigualdad, así como la exclusión de ciertos grupos o sectores de este servicio, impiden pasar a un segundo momento evaluativo

- La calidad de la deliberación en el procedimiento y el uso "eficiente" de los recursos del CrowdLaw no puede legitimar por si sola la decisión, si aquella no ha sido resultado de la inclusión de las voces de los potenciales afectados

De esta manera, no basta con que el CrowdLaw posibilite acercarse a la idea de un gobierno abierto a través del uso de las nuevas tecnologías, sino que resulta relevante la cuestión sobre quiénes participan y en qué condiciones participan. El espacio digital que se presenta como un espacio democrático, que pretende igualar las condiciones de participación, arrastra muchas veces las desigualdades y las brechas sociales. Así, como advierte Tello-Arista (2016), cualquier proyecto de CrowdLaw que busque ser efectivo y productivo debe asumir los desafíos identificados para incorporarlos en su diseño, sobre todo en lo relativo a las brechas de participación. Sin embargo, consideramos que los retos que anotamos deben ser observados no solo en el proceso de diseño, sino que deben extenderse a las etapas de implementación y evaluación de los mecanismos del CrowdLaw, si lo que busca es mejorar las democracias, evitando traducir sus defectos al espacio digital.

\section{Conclusiones}

CrowdLaw surge como una propuesta innovadora para la mejora de la calidad y la legitimidad de las decisiones públicas, promoviendo la participación ciudadana en las diversas etapas de la producción de normas y políticas a través de las nuevas tecnologías. Esta propuesta ha sido recibida en diferentes 
partes del globo, promoviéndose diversas iniciativas orientadas a materializar sus objetivos.

La democracia deliberativa, como horizonte normativo adoptado por estas medidas, requiere una traducción de sus condiciones y criterios evaluativos al espacio digital, lo que implica analizar a su vez las condiciones sociales que posibilitan la participación ciudadana en dicho espacio. Es así como surgen nuevas implicancias, derivadas de que en este caso la participación política depende del acceso a las nuevas tecnologías y a Internet como punto de partida. Lo complejo de esta situación es que, incluso garantizándose su acceso, surgen otras cuestiones como la relativa a la educación para el uso de estas nuevas tecnologías. Ello evitaría que las decisiones que se tomen sean resultado de la participación de ciertos grupos que tienen la capacidad de acceder y hacer un uso adecuado de las plataformas y herramientas de CrowdLaw, y no necesariamente de la participación de los potencialmente afectados por la medida, como exige la democracia deliberativa, quienes pueden verse excluidos por falta de acceso o de capacitación.

Pese a ello, es necesario destacar como un avance importante en materia de participación ciudadana, que los mecanismos de CrowdLaw estén orientados a la producción de inteligencia colectiva y promuevan la interacción entre ciudadanos, representantes y funcionarios públicos, lo que puede contribuir a la formación de una democracia más exigente que pueda hacer frente a la crisis de representación actual. Consideramos que, para su eficacia, los retos advertidos exigen el cierre de las brechas digitales como condición fundamental para una democracia deliberativa basada en las nuevas tecnologías.

\section{Referencias}

Aitamurto, T. (2015). Crowdsourcing as a Knowledge-Search Method in Digital Journalism. Digital Journalism, 4(2), 280-297. doi:10.1080/21670811.2015.1034807

Aitamurto, T. y Landemore, H. (2015). Five design principles for crowdsourced policymaking: Assessing the case of crowdsourced off-road traffic law in Finland. Journal Social Media for Organizations, 2(1), 1-19.

Aitamurto, T. y Landemore, H. (2016). Crowdsourced Deliberation: The Case of the Law onOff-Road Traffic in Finland. Policy \& Internet, 8(2), 174-196.

Alsina, V. y Martí, J. L. (2018). The Birth of the CrowdLaw Movement: Tech-Based Citizen Participation, Legitimacy and the Quality of Lawmaking. Analyse \& Kritik, 40(2), 337-358.

Asamblea General de la ONU. (2011). Informe del Relator Especial sobre la promoción y protección del derecho a la libertad de opinión y expresión Frank Le Rue. A/ $H R C / 17 / 27$. 
Beetham, D. (1991). The Legitimation of Power. Palgrave.

Bohman, J. (2016). La madurez de la democracia deliberativa. Co-herencia, 13(24), 105143.

Brabham, D. C. (2013). Crowdsourcing. MIT Press.

Brito, J. (2008). Hack, Mash y Peer. Crowdsourcing Government Transparency. The Columbia Science and Technology Law Review, 119(9), 119-157.

Buchanan, A. (2002). Political Legitimacy and Democracy. Ethics, 112(4), 689-719.

Christiano, T. (1996). The Rule of the Many. Westview Press.

Cohen, J. (1997). "Procedure and Substance in Deliberative Democracy". In J. Bohman, \& W. Rehg, Deliberative Democracy: Essays on Reason and Politics (pp. 407-438). The Massachusetts institute of Technology Press.

Dahlberg, L. (2007). The Internet, deliberative democracy, and power: Radicalizing the public sphere. International Journal of Media \& Cultural Politics, 3(1), 47-64.

De Moraes, A. (2019). Assessing the Effects of CrowdLaw Initiatives: Experiences from Latin America. In 2019 Sixth International Conference on eDemocracy \& eGovernment (ICEDEG), 365-366.

Elster, J. (1998). Deliberative democracy. Cambridge: Cambridge University Press.

Ess, C. (2018). Democracy and the Internet: A retrospective. Javnost, 25(1-2), 93-101.

Ercan, S. A., Hendriks, C. M., \& Dryzek, J. S. (2019). Public deliberation in an era of communicative plenty. Policy \& politics, 47(1), 19-36.

Fishkin, J. S., Senges, M., Donahoe, E., Diamond, L., y Siu, A. (2018). Deliberative polling for multistakeholder Internet governance: considered judgments on access for the next billion. Information, Communication \& Society, 21(11), 1541-1554.

Garnelo, M. (2019). Measuring CrowdLaw: Developing Indicators to Measure the Impact of Participatory Processes on the Quality of Policy-making in Madrid (Doctoral dissertation, Harvard University).

Gimmler, A. (2001). Deliberative democracy, the public sphere and the Internet. Philosophy \& Social Criticism, 27(4), 21-39.

Giuffré, C. I. (2018). Democracia deliberativa y surgimiento del constitucionalismo dialógico. Ética y Discurso, 1(3), 35-60.

GovLab. (2018). CrowdLaw Manifesto. Recuperado el 04 de marzo de.2020 de: https:// manifesto.crowd.law/es/

GovLab. (2018). Catalog. Recuperado el 04 de marzo de 2020 de: https://catalog.crowd. law/index.html

GovLab. (2018). About CrowdLaw. Recuperado el 04 de marzo de 2020 de: https:// catalog.crowd.law/about.html

Gutmann, A. y Thompson, D. F. (2004). Why deliberative democracy? Princeton University Press.

Hopenhayn, M. (2006). Desigualdades Sociales y Derechos Humanos: Hacia un pacto de Protección Social. Reunión de Expertos sobre Población, Desigualdades y Derechos Humanos. CEPAL. Recuperado el 04 de marzo de 2020 de: https://www.cepal.org/ sites/default/files/events/files/hopenhaynm.pdf

Internet World Stats. (2020, marzo 03). Internet World Penetration Rates by Geographic Regions - 2020 Q1. Retrieved from Internet World Stats: https://www. Internetworldstats.com/stats.htm\#links

Kittur, A., Chi, E. H. y Suh, B. (2008). Crowdsourcing user studies with Mechanical Turk. Proceedings of the SIGCHI Conference on Human Factors in Computing Systems (p. 453-456). ACM. 
Klein, M. (2011). How to Harvest Collective Wisdom for Complex Problems: An Introduction to the MIT Deliberatorium. MIT Center for Collective Intelligence.

Landemore, H. (2020). Open Democracy: Reinventing Popular Rule for the Twenty-First Century. Princeton University Press.

Levy, P. (2004). Inteligencia colectiva: por una antropología del ciberespacio. Biblioteca virtual en salud.

McKay, S. y Tenove, C. (2020). Disinformation as a threat to deliberative democracy. Political Research Quarterly, 73.

Martí, J. L. (2011). La paradoja de las precondiciones de la democracia deliberativa en Nino. In L. Garíca-Jaramillo, La democracia deliberativa a debate (p. 41-54). Universidad EAFIT.

Meier, P. (2011). Do 'Liberation Technologies' Change the Balance of Power between Repressive States and Civil Society? University of Medford, Fletcher School of Law and Diplomacy. University of Medford.

Nino, C. S. (1996). La constitución de la democracia deliberativa. Barcelona: Gedisa.

Noveck, B. S. (2018). CrowdLaw: Collective Intelligence and Lawmaking. Analyse \& Kritik, 40(2), 359-380.

Rawls, J. (1993). Political Liberalism. Columbia University Press.

Rosgaby Medina, K. (2020). Estadísticas de la situación digital de Colombia en el 2019 y 2020. Recuperado el 04 de marzo de 2020 de: https://branch.com.co/marketingdigital/estadisticas-de-la-situacion-digital-de-colombia-en-el-2019-y-2020/

Sgueo, G. (2020). Using Technology to 'Co-Create'EU Policies. European Parliamentary Research Service. Recuperado el 04 de marzo de 2020 de: https://ssrn.com/ abstract $=3521764$

Tello Arista, I. (2016). Measuring CrowdLaw: Developing Indicators Improving Policy Legitimacy through Crowdsourcing Legislation (Master's dissertation, New York University). http://ecas.issuelab.org/resources/29679/29679.pdf

Thompson, D. (2019). Teoría democrática deliberativa y ciencia política empírica. Estudios Políticos (Universidad de Antioquia)(56), 270-313.

Unión Internacional de Telecomunicaciones. (2020). Inclusión digital para todos. Recuperado el 04 de marzo de 2020 de: https://www.itu.int/es/mediacentre/ backgrounders/Pages/digital-inclusion-of-all.aspx

Waldron, J. (2005). Derecho y Desacuerdos. Marcial Pons.

Weber, M. (1964). The Theory of Social and Economic Organization. Michigan: Talcott Parsons, Free Press.

Witschge, T. (2004). Online deliberation: Possibilities of the Internet for deliberative democracy. Democracy online: The prospects for political renewal through the Internet, 109-122. 